\title{
MAKER: From 2-D Projective Geometry to 3-D Object Recognition and 3-D Printing Processes for High School Students
}

\section{Mr. Bart Taylor M.Ed., A\&M Consolidated High School}

A Dedicated career and technology teacher with fifteen years of experience in the classroom. Offer a proven track record of commended performance in teaching, and leadership, with a passion for education and a commitment to continually pursue student, school and district success. Experience includes classroom teaching, motivational speaking, district curriculum and instructional coaching, professional development planning/presenting, high school webpage administrator, TIVA Board Member, e2it3 President, robotics/engineering club head sponsor, Gay Straight Alliance sponsor and SkillsUSA Texas District 7 Director.

Currently working on an Ed.D. in Curriculum and Instruction at Texas A\&M University in the Department of Teaching, Learning and Culture in College Station, TX. Plans are to pursue furthering a career in education at the district or university level as well as increasing experience in public and motivational speaking.

\section{Dr. Sheng-Jen "Tony" Hsieh, Texas A\&M University}

Dr. Sheng-Jen ("Tony") Hsieh is a Professor in the Dwight Look College of Engineering at Texas A\&M University. He holds a joint appointment with the Department of Engineering Technology and the Department of Mechanical Engineering. His research interests include engineering education, cognitive task analysis, automation, robotics and control, intelligent manufacturing system design, and micro/nano manufacturing. He is Director of the Rockwell Automation laboratory at Texas A\&M University, a stateof-the-art facility for education and research in the areas of automation, control, and automated system integration. He also serves as Director of an NSF Research Experiences for Teachers (RET) program in the area of Mechatronics, Robotics, and Industrial Automation.

\section{Prof. Dezhen Song}




\title{
MAKER: From 2D Projective Geometry to 3D Object Recognition and 3D Printing Processes for High-School Students
}

\begin{abstract}
This paper describes the process on learning to apply the concepts of Projective Geometry by the use cameras to capture images of a scene and using those images to calculate the location of a given object within a defined space. Once the concepts of 2D Projective Geometry are understood, Autodesk's 123D Catch software will be used to reconstruct 3D objects in a planar environment. The process will consist of the use of digital cameras to capture varying angles and distances of a pinpointed object so that projective geometry calculations can create a 3D model of the given object. The 123D Catch demonstration will help support concepts in Computer Vision and SLAM programming.
\end{abstract}

\section{Motivation}

The world today is being captured by digital cameras that are incorporated into so many of our everyday devices. From cell phones and tablets, to glasses and watches, these digital cameras are quickly becoming our pair of "digital eyes." The interpretation of these visual images by computers opens a pathway to the field of study called Computer Vision. The concept of Computer Vision was developed by utilizing computers to interpret digital images, combined with the focal length of the camera, to understand and reconstruct $3 \mathrm{D}$ objects and scenes from 2D planar images. The guiding concept of Computer Vision comes from the interpretation of projective geometry in images by using Simultaneous Location and Mapping algorithms, also known as SLAM. Programs such as AutoDesk 123D Catch and Adobe Photoshop take advantage of SLAM programming in order to reconstruct an object in 3D derived from 2D images or rescaled or re-proportioned images based upon the usage of projective transformation. Computer Vision combines Science, Technology, Engineering and Mathematics into a study with practical applications in robotics, humanoid interactive recognition, bio-technology, video gaming and 3D mapping.

\section{Keywords}

3D Modeling, affine transformation, Computer Vision, Projective Geometry, projective transformation, similarity transformation, SLAM Algorithm

\section{Pre-Requisite Knowledge}

Students should have an understanding of geometry, 2D vs 3D views and basic camera techniques. Students should have a clear understanding of a technology system and its basic parts.

\section{Learning Objectives}

- After this activity, students should be able to: 
- Identify and apply the concepts of Projective Geometry through the use of digital cameras.

- Reconstruct Object location within a defined plane by using Projective Transformation.

- Reconstruct a 3D object through the use of digital cameras.

- Collect and Organize, Analyze and Present Data

\section{Materials List}

- Digital camera (Cell Phone cameras can be utilized.)

- Rulers

- Computer with Autodesk 123D Catch

- Optional: Motion Camera such as a GoPro

- Optional: 3D Printer

\section{Introduction / Motivation}

Digital cameras are everywhere. You can find digital cameras in computers, laptops, tablets, cell phones, watches and more. Digital cameras are also all over the world in cityscapes, traffic lights buildings and more. Access the following video for an idea of how digital cameras are in a growing field of Computer Vision.

\section{https://www.youtube.com/watch?v=thgNA6nivWU}

If digital cameras can become our digital "eyes", what kinds of applications can we see this technology being used? Biotechnology ad robotics? Please access the following video for inspiration in Computer Vision.

\section{https://www.youtube.com/watch?v=VvkIdCK1L54}

Computer vision is the discipline that studies how to reconstruct, interpret, and understand a 3D scene from its 2D images in terms of the properties of the structures presented in the scene. Projective geometry is the study of geometric properties that are invariant under projective transformations such as Similarity Transformation, Affine Transformation and Projective Transformation.

There are several methods that can be used in reconstructing an object in 3D from digital pictures. The method that this research follows is with the free software provided by AutoDesk, 123D Catch. To be successful, one must start with the knowledge and background of 3D modeling and projective geometry. Understanding of Similarity, Affine and Projective Transformation is vital.

Similarity Transformation - Two geometrical objects are called similar if they both have the same shape, or one has the same shape as the mirror image of the other. More precisely, one can be obtained from the other by uniformly scaling (enlarging or shrinking), possibly with additional translation, rotation and reflection. This means that either object can be rescaled, repositioned, and reflected, so as to coincide precisely with the other object. 


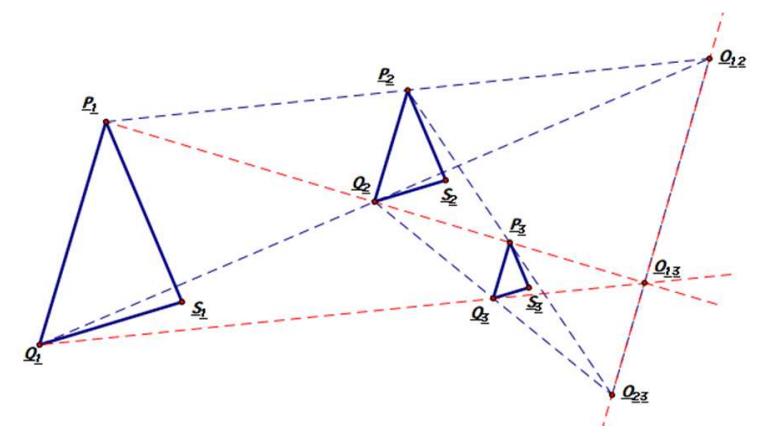

Figure 1. Similarity Transformation

Affine Transformation - A mapping from one vector space to another, consisting of a linear part, expressed as a matrix multiplication, and an additive part, an offset or translation.

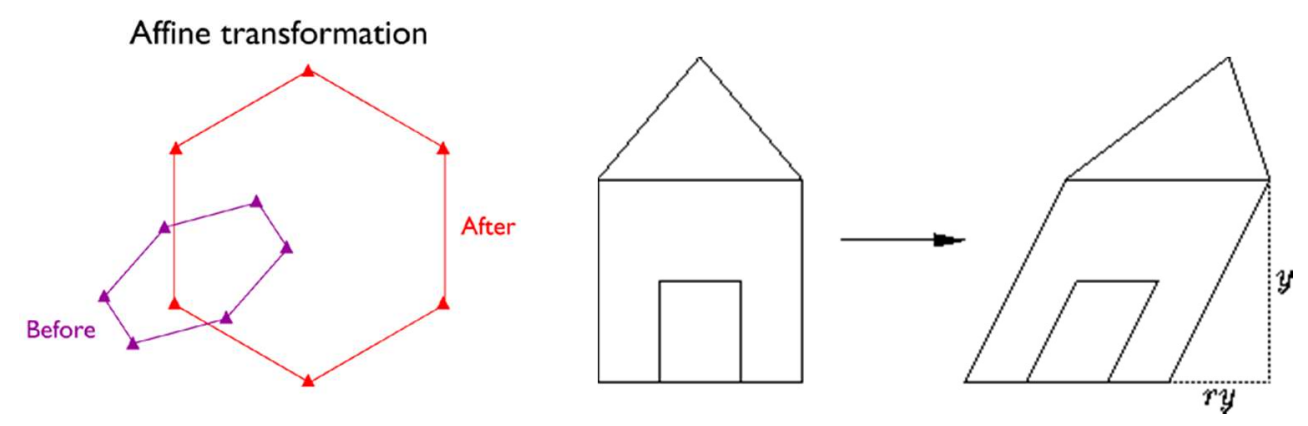

Figure 2. Affine Transformation

Projective Transformation - A transformation of space that sends points into points, lines into lines, planes into planes, and any two incident elements into two incident elements.
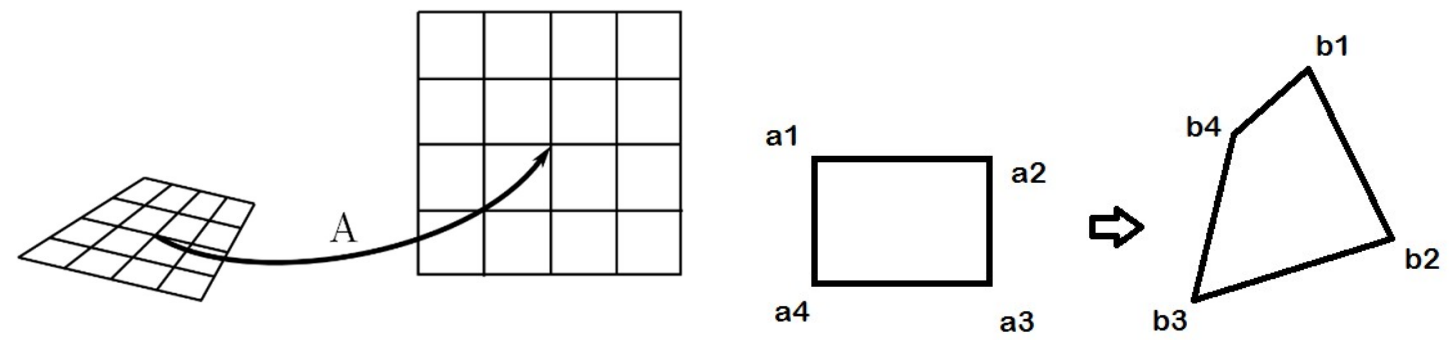

Figure 3. Projective Transformation 


\section{SLAM Algorithm}

Simultaneous localization and mapping (SLAM) is a technique used by robots and autonomous vehicles to build up a map within an unknown environment (without a priori knowledge), or to update a map within a known environment (with a prior knowledge from a given map), while at the same time keeping track of their current location.
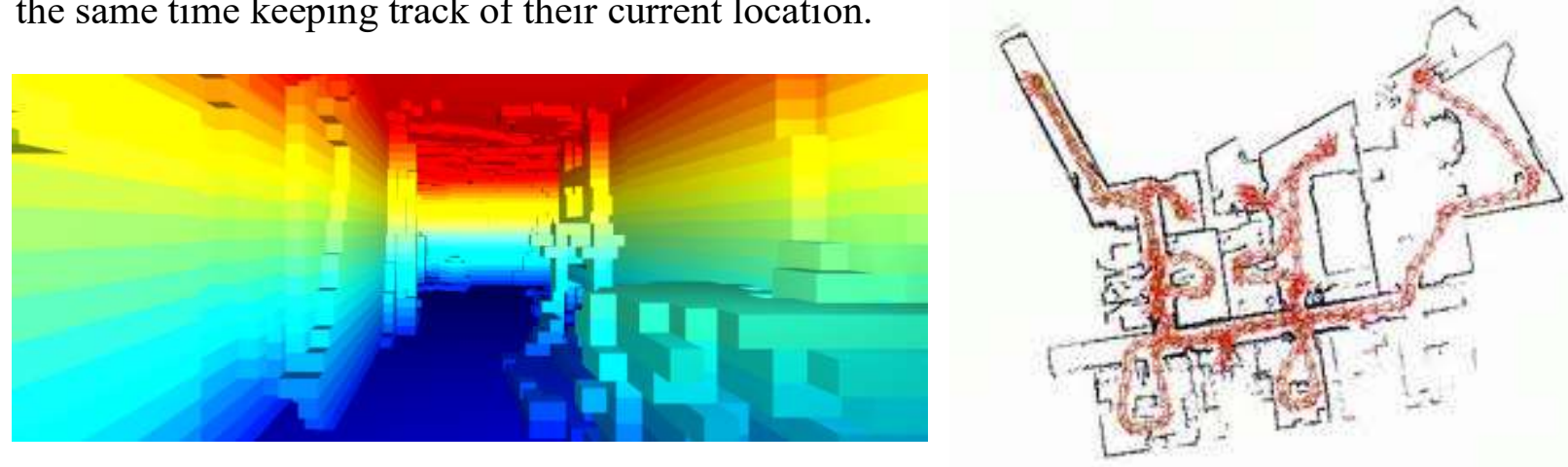

Figure 4. SLAM Algorithm

Camera Calibration is another key aspect in SLAM programming and is utilized within the 123D Catch program. To calibrate a camera, the technique only requires the camera to observe a planar pattern shown at a few (at least two) different orientations.

Acquiring digital images of the presented object requires proper planning and procedure. A higher quality digital image will result in a better 3D capture, however images should not be altered from their original file after taking the images. Proper lighting is necessary. There should be no directional lighting that would result in shadowing and the level of light should be averaged so as not to offset the white balance. A series of a minimum of 20., maximum of 70 , sequential photographs should be taken in small increments around the subject. Following proper image acquisition, the files should be uploaded and processed by 123D Catch. During this process, the power of cloud computing and shared processing will allow 123D catch to render a 3D model derived from the series of photographs. From the camera calibration, to the knowledge of the focal length in parallel with projective transformation, the software can calculate a $3 \mathrm{D}$ model based upon the series of images. The model is rendered into the software for further editing. From there, the model can be refined, scaled and printed in $3 \mathrm{D}$.

\section{Review of the Literature}

Over the last decade, there has been a push to make Science, Technology, Engineering, and Mathematics, or STEM, education a priority in K-12 schools in order to help keep the United States globally competitive in science, technology, medicine and engineering. Many have supported STEM initiatives including President Obama in his 2012 State of the Union Address when he stated, "Think about the America within our reach. A country that leads the world in educating its people. An America that attracts a new generation of high tech manufacturing and high paying jobs" (as cited in Avery, Z., 2013, p. 56). More recently the maker movement has been incorporated into the classic Science, Technology, Engineering and Math models. The 
maker movement culture emphasizes learning by doing, construction, and innovation. The trend might be new, but the model is not. Doughtery (2012) explains how "a century ago, psychologist and education reformer John Dewey extolled the virtues of learning by doing, and contemporary science of the brain confirms the importance of tactical engagement and of using our hands in the learning process" (p. 12).

The maker movement has come about in part because of people's need to engage passionately with objects in ways that make them more than just consumers. Makers, at their core are enthusiasts, such as those engaged in the early days of the computer industry in Silicon Valley. The core of the maker movement parallels the needs of STEM education and is expanding into education, business and government. The Maker movement has a simple formula, one based entirely on talking to people who make things, seeing what those people do, and nurturing the diversity of ideas that come together in a community space. Providing students room for inquiry and innovation in science creates students who have a stronger background in STEM topics but also in problem solving and collaboration, all of which are imperative 21 st century skills. Software such as the 123D catch provided by Autodesk adheres to the Maker movement and supports the do-it-yourself culture.

\section{Vocabulary / Definitions}

\begin{tabular}{|l|l|}
\hline \multicolumn{1}{|c|}{ Word } & \multicolumn{1}{c|}{ Definition } \\
\hline 3D Modeling & Three-Dimensional representation of an object. \\
\hline Affine Transformation & $\begin{array}{l}\text { A mapping from one vector space to another, consisting of a } \\
\text { linear part, expressed as a matrix multiplication, and an additive } \\
\text { part, an offset or translation. }\end{array}$ \\
\hline Projective Geometry & $\begin{array}{l}\text { Computer vision is the discipline that studies how to reconstruct, } \\
\text { interpret, and understand a 3D scene from its 2D images in terms } \\
\text { of the properties of the structures presented in the scene. }\end{array}$ \\
\hline Projective & $\begin{array}{l}\text { The study of geometric properties that are invariant } \\
\text { under projective transformations. }\end{array}$ \\
\hline Sransformation & $\begin{array}{l}\text { A transformation of space that sends points into points, lines into } \\
\text { lines, planes into planes, and any two incident elements into two } \\
\text { incident elements. }\end{array}$ \\
\hline SLAM Algorithm & $\begin{array}{l}\text { Two geometrical objects are called similar if they both have the } \\
\text { same shape, or one has the same shape as the mirror image of the } \\
\text { other. More precisely, one can be obtained from the other by } \\
\text { uniformly scaling (enlarging or shrinking), possibly with } \\
\text { additional translation, rotation and reflection. This means that } \\
\text { either object can be rescaled, repositioned, and reflected, so as to } \\
\text { coincide precisely with the other object. }\end{array}$ \\
\hline $\begin{array}{l}\text { Simultaneous localization and mapping (SLAM) is a technique } \\
\text { used by robots and autonomous vehicles to build up a map within } \\
\text { an unknown environment (without a priori knowledge), or to } \\
\text { update a map within a known environment (with a prior }\end{array}$ \\
\hline
\end{tabular}




\section{Before the Activity}

Please make sure that Autodesk 123D Catch is installed in your computer lab as well as the 123D Printing Utility. This activity is divided into two weeks. Depending on the students' preparation and their progress, it may take less time. The activity requires students to be in groups of two to three. Students will be using digital cameras to acquire image data so that an object can be reconstructed in 3D. Please plan to have time for uploading of the images to be processed. Students will also spend time online with video tutorials. Below is the table that provides the scope and sequence of the activity.

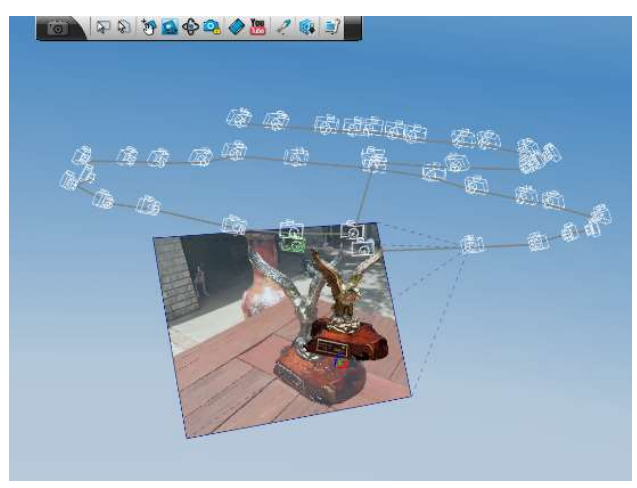

Figure 5. AutoDesk 123D Catch screenshot

Watch the following tutorial videos on AutoDesk's 123D Catch:

1. Tips for a successful shoot: https://www.youtube.com/watch?v=7TfXXJXDsXw

2. Starting your first project: https://www.youtube.com/watch? $\mathrm{v}=9 j \mathrm{IUU} 0 \mathrm{vJdAHs}$

3. Navigating your 3D scene: $h$ ttps://www.youtube.com/watch? $v=4 \mathrm{GvbO} z \mathrm{z} 0 \mathrm{mpE}$

4. Creating animations: https://www.youtube.com/watch? $\mathrm{v}=\mathrm{YHbcqBFHf} 1 \mathrm{w}$

5. Shooting and image upload: https://www.youtube.com/watch?v=WZB7RUFS2fo

6. Mesh details: https://www.youtube.com/watch?v=zlarVKBAEtM

7. Manual stitching: $h$ ttps://www.youtube.com/watch? $\mathrm{v}=5 \mathrm{yPt} 66 \mathrm{D} 1 \mathrm{~S} 2 \mathrm{E}$

8. Reference points: https:/www.youtube.com/watch? v=q1ByqizHeEE

9. Custom coordinate system: https://www.youtube.com/watch?v=yD1FV-cFzYE 


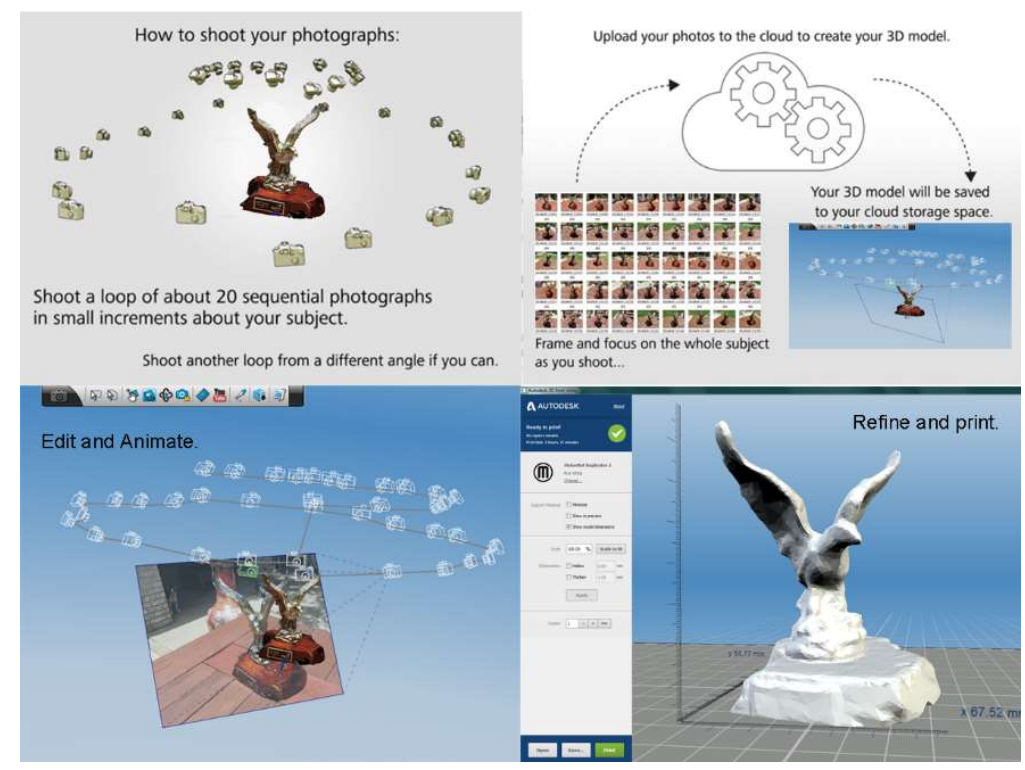

Figure 6. AutoDesk 123D Catch screenshot

\section{Data Acquisition}

Students should work in assigned groups to take their initial digital images of the assigned objects. They should set up their own technique and lighting if they address it. Save all images for uploading.
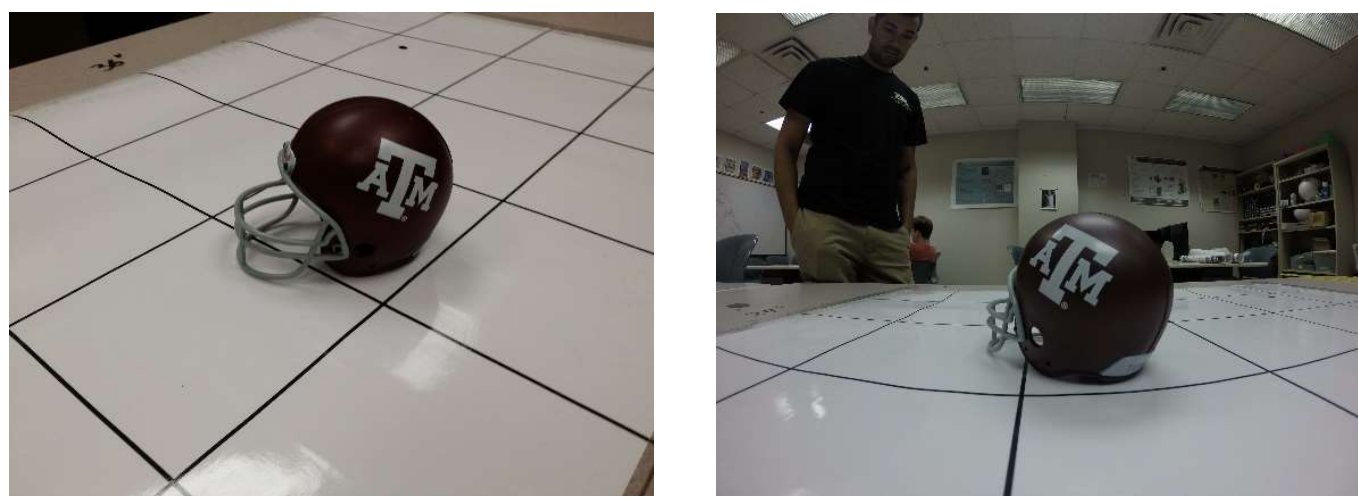

Figure 7. Data Acquisition

\section{Image Processing}

Students should will upload their initial digital images of the assigned objects to be processed in 123D Catch. Students will spend the time editing the object, rendering an animation of the object and exporting their work. 


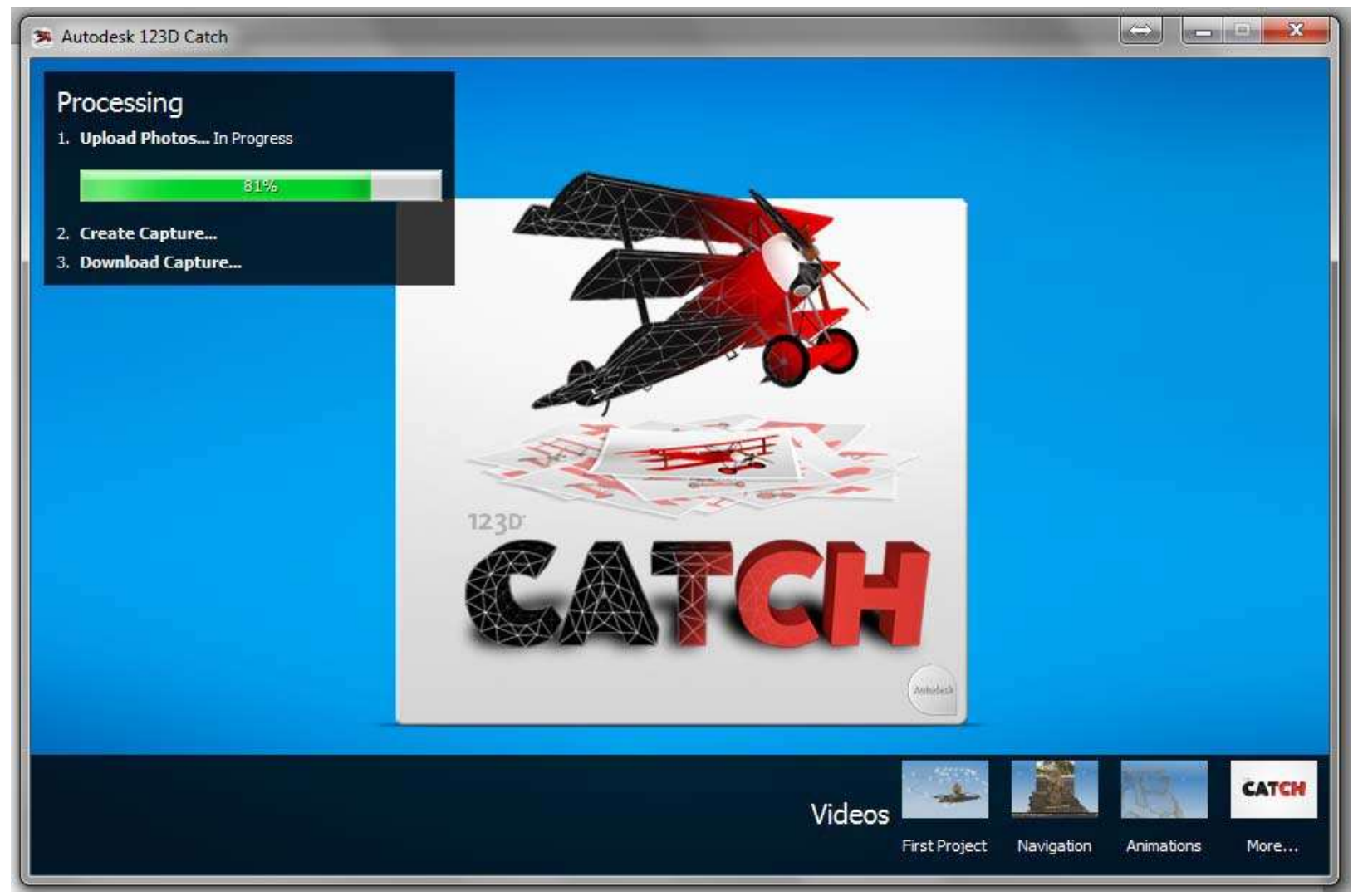

Figure 8. 123D Catch Image Processing

\section{D Modeling}

Watch the following tutorial videos on AutoDesk's 123D Catch:

1. Turn photographs into 3D models: https:/www.youtube.com/watch? $\mathrm{v}=9 \mathrm{nRO}$ RKyK4bk

2. Prepare your capture for 3D printing: https://www.youtube.com/watch? $\mathrm{v}=\mathrm{rHvyidObpPk}$

3. 3D print your capture with a web service: https://www.youtube.com/watch? $\mathrm{v}=6 \mathrm{VHR}-$ JGc8wI

Students should now work to refine their digital images of the assigned objects and upload it to be process in 123D Catch. Students should spend the time editing the object, rendering an animation of the object and exporting their work.

Optional: Students will prepare their objects for 3D Printing. Student ready to begin printing should print. This process can be time consuming. 


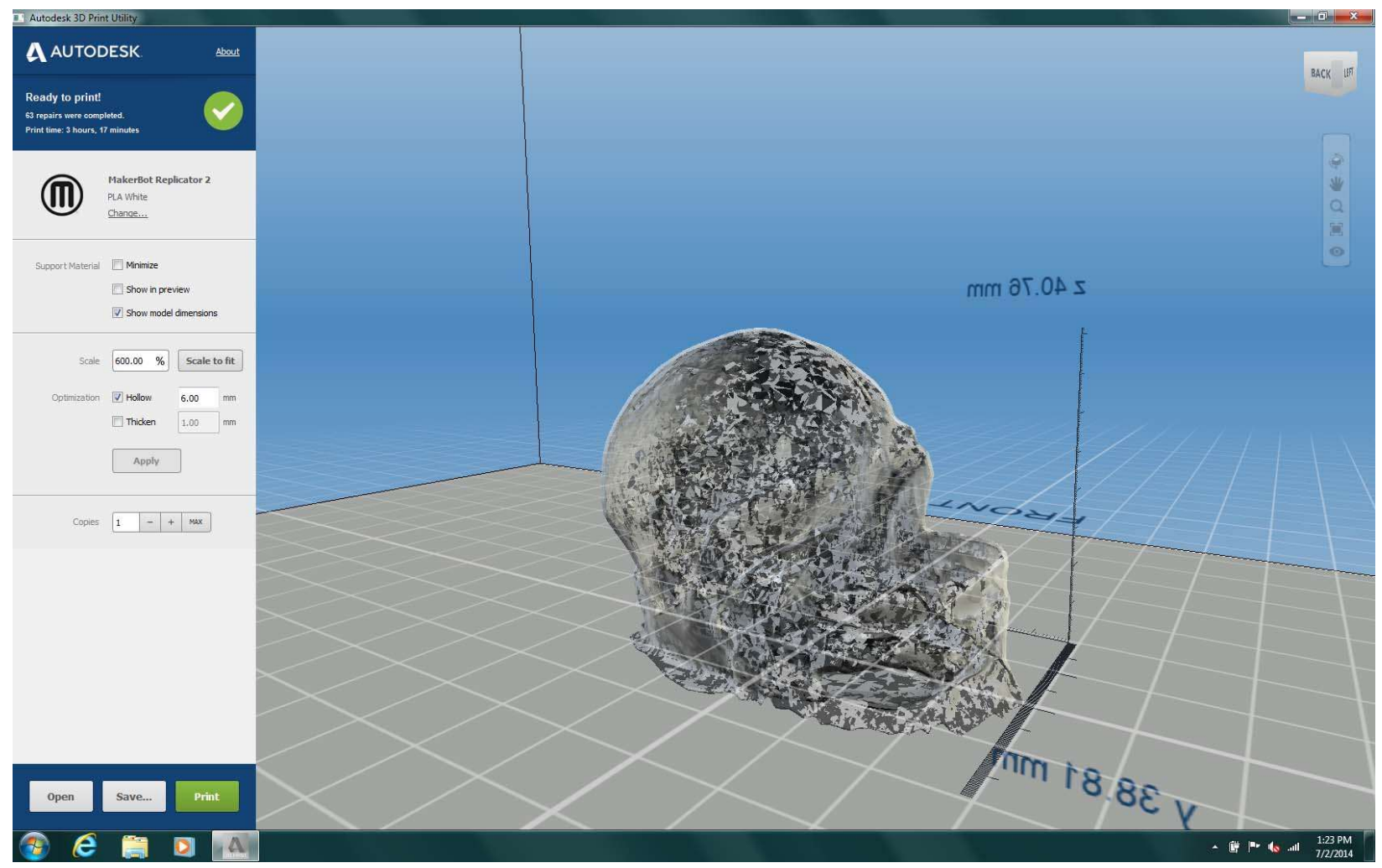

Figure 9. 3D Image Modeling and printing preparation

\section{Results}

Students that completed the activity were assessed to have an average grasp of Computer Vision, but full understanding of applying 3D and projective Geometry concepts. The benefit was a continued spark into 3D design and interest in their own creativity that led to an increased desire to learn 3D printing technologies.

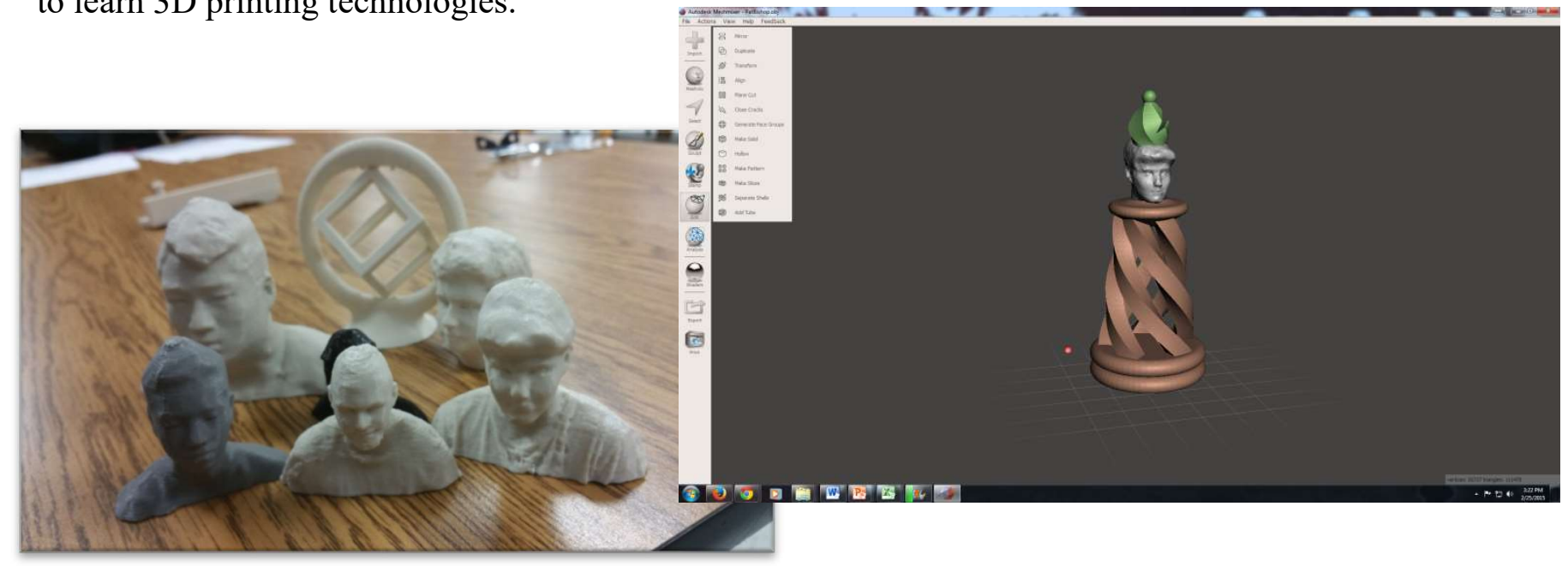

Figure 10. 3D Image Modeling Results 


\section{Conclusion}

Computer Vision is a fascinating field of study that encompasses $40 \%$ of all Computer Science Research today. By utilizing and applying the theories of Computer Vision, SLAM Algorithms and Projective Geometry while having the ability to reconstruct and object in 3D, programs such as AutoDesk's 123D Catch are making huge waves in the frontier of Computer Vision. Digital cameras, in combination with 3D printing, opens new pathways in research, analysis and even data acquisition. The utilization of this research can be applied into many real world applications, such as robotics and automation, location mapping, search and rescue, hazard detection and avoidance, 3D video gaming and gesture recognition.

\section{Contributors}

Bart Taylor, A\&M Consolidated High School, College Station, TX Dr. Dehzen Song, Texas A\&M University Computer Science Engineering, College Station, TX

\section{Acknowledgements}

This material is based upon work supported by the Research Experiences for Teachers in Mechatronics, Robotics, and Industrial Automation Program under National Science Foundation Grant No. 1300779. Any opinions, findings, and conclusions or recommendations expressed in this material are those of the author(s) and do not necessarily reflect the views of the National Science Foundation.

\section{References}

1. 3D for Everyone. (n.d.). Retrieved from http://www.sketchup.com/

2. Dezhen Song. (n.d.). Retrieved from http://faculty.cs.tamu.edu/dzsong/

3. Dougherty, D. (2012). The maker movement. Innovations, 7(3), 11-14.

4. Hartley, R., \& Zisserman, A. (2003). Multiple view geometry in computer vision. Cambridge, UK: Cambridge University Press.

5. Learn how to use 123d Catch. (n.d.). Retrieved from http://www.123dapp.com/howto/catch

6. Peppler, K., Maltese, A., Keune, A., Chang, S., Regalla, L., \& Initiative, M. E. (2015). Survey of Makerspaces, Part II. Open Portfolios Maker Education Initiative.

7. Teach Engineering. (n.d.). Retrieved from http://www.teachengineering.org/

8. Technische Universität München. (n.d.). Retrieved from http://vision.in.tum.de/data/datasets/rgbddataset 